\title{
What is Computational Intelligence and where is it going?
}

\author{
Włodzisław Duch \\ Department of Informatics, Nicolaus Copernicus University, Grudziądzka 5, Toruń, Poland, and \\ School of Computer Engineering, Nanyang Technological University, Singapore
}

\begin{abstract}
What is Computational Intelligence (CI) and what are its relations with Artificial Intelligence (AI)? A brief survey of the scope of CI journals and books with "computational intelligence" in their title shows that at present it is an umbrella for three core technologies (neural, fuzzy and evolutionary), their applications, and selected fashionable pattern recognition methods. At present CI has no comprehensive foundations and is more a bag of tricks than a solid branch of science. The change of focus from methods to challenging problems is advocated, with CI defined as a part of computer and engineering sciences devoted to solution of non-algoritmizable problems. In this view AI is a part of $\mathrm{CI}$ focused on problems related to higher cognitive functions, while the rest of the CI community works on problems related to perception and control, or lower cognitive functions. Grand challenges on both sides of this spectrum are addressed.
\end{abstract}

\section{Introduction}

What exactly is Computational intelligence (CI)? How is it related to other branches of computer science, such as artificial intelligence (AI), classification, cognitive informatics, connectionism, data mining, graphical methods, intelligent agents and intelligent systems, knowledge discovery in data (KDD), machine intelligence, machine learning, natural computing, parallel distributed processing, pattern recognition, probabilistic methods, soft computing, multivariate statistics, optimization and operation research? This is a very confusing issue, hotly debated, but with no consensus in sight. Computational intelligence became a new buzzword that means different things to different people.

Branches of science are not defined, but slowly develop in the process of sharing and clustering of common interests. Even well-established sciences have problems with clear definition: for example, one of the best definition of physics is "physics is what physicist do". Herbert Simon, one of the AI fathers, answered the question "What is artificial intelligence?" writing "We define it in terms of the tasks that are done" [1]. Computational Intelligence experts focus on problems that are difficult to solve using artificial systems, but are solved by humans and some animals, problems requiring intelligence. Specific interests also focus on methods and tools that are applicable to this type of problems. Starting with seminal papers, special sessions, growing into separate conferences and specialized journals, different branches of CI evolve in many directions, frequently quite far from original roots and inspirations. New communities are 
formed and need to establish their identity by defining borders distinguishing them from other scientific communities.

Artificial Intelligence (AI) was the first large scientific community, established already in the mid 1950s, working on problems that require intelligence to be solved. Its evolution has been summarized in the 25th anniversary issue of the AI Magazine by Mackworth [2]: "In AI's youth, we worked hard to establish our paradigm by vigorously attacking and excluding apparent pretenders to the throne of intelligence, pretenders such as pattern recognition, behaviorism, neural networks, and even probability theory. Now that we are established, such ideological purity is no longer a concern. We are more catholic, focusing on problems, not on hammers. Given that we do have a comprehensive toolbox, issues of architecture and integration emerge as central."

IEEE Computational Intelligence Society defines its subjects of interest as neural networks, fuzzy systems and evolutionary computation, including swarm intelligence. The approach taken by the journals and by the book authors is to treat computational intelligence as an umbrella under which more and more methods are slowly added. A good definition of the field is therefore impossible, because different people include or exclude different methods under the same CI heading. Chess programs based on heuristic search are already in the superhuman computational intelligence category, but they do not belong to CI defined in such a way. In the early days of CI some experts tried to explicitly exclude problems requiring reasoning. Take for example this definition: "A system is computationally intelligent when it: deals only with numerical (low level) data, has a pattern recognition component, and does not use knowledge in the AI sense" [3].

As in the case of Artificial Intelligence the need to create strong identity by emphasizing specific methods defining Computational Intelligence as a field should be replaced by focus on problems to be solved, rather than hammers. Below some remarks on the current state of $\mathrm{CI}$ are made, based on analysis of journals and books with "computational intelligence" in their title. Then a new definition of CI is proposed and some remarks are made on what should the computational intelligence field really be in future. Finally grand challenges to computational intelligence are discussed.

\section{CI journals}

The name "Computational Intelligence" has been used for over 20 years, although only recently it has gained a widespread popularity and somewhat different flavor. There are already at least 10 journals with "Computational Intelligence" in the title and the number of journals with "intelligent" or "intelligence" in the title is far greater.

The quarterly journal Computational Intelligence. An International Journal (Blackwell Publishing, since 1984) is the oldest among CI journals. It is focused on typical artificial intelligence problems, related to higher cognition: logic, reasoning, planning, complex agents, language understanding, rule-based machine learning and reinforcement learning. In the description of the journal it is clearly stated that: "This leading international journal promotes and stimulates research in the field of artificial intelligence (AI).... The journal is designed to meet the needs of a wide range of AI workers in academic and industrial research." The main focus areas include AI applications in 
entertainment, software engineering, computational linguistics, web intelligence, business, finance, commerce and economics. Unfortunately this journal makes an impression that computational intelligence is just another name for artificial intelligence.

The Journal of Computational Intelligence in Finance (Finance \& Technology Publishing, since 1993) was focused on the financial applications of CI predictive methods, but seems to have vanished by now.

The International Journal of Computational Intelligence and Organizations (Lawrence Erlbaum Associates, since 1996) is a quarterly journal focusing on theories, methods and applications of computational intelligence in organizations. This journal "publishes original, high-quality articles dealing with the design, development, implementation and management of neural networks, genetic algorithms, fuzzy logic, uncertain reasoning techniques, and related machine learning methods as they apply to organizations. Application of alternative techniques and comparisons to other artificial intelligence models, nonparametric statistics, and decision trees are encouraged. The emphasis is on how computational intelligence is being applied to decision making and problem solving in organizations." Note that this journal (unfortunately conspicuously absent in the Internet) encourages comparisons of results with alternative techniques that may be used to solve the same problem.

The Journal of Advanced Computational Intelligence and Intelligent Informatics (Fuji Technology Press, since 1997) is published bimonthly. This journal focuses on "the synergetic integration of neural networks, fuzzy logic and evolutionary computation", and building intelligent systems for industrial applications. Except for the standard fuzzy, neural and evolutionary computation triad, "hybrid systems, adaptation and learning systems, distributed intelligent systems, network systems, multi-media, human interface, biologically inspired evolutionary systems, artificial life, chaos, fractal, wavelet analysis, scientific applications and industrial applications" are also mentioned.

The International Journal of Computational Intelligence and Applications (World Scientific, since 2001) is "dedicated to the theory and applications of computational intelligence (artificial neural networks, fuzzy systems, evolutionary computation and hybrid systems). The main goal of this journal is to provide the scientific community and industry with a vehicle whereby ideas using two or more conventional and computational intelligence based techniques could be discussed." Areas include neural, fuzzy and evolutionary computation, pattern recognition, hybrid intelligent systems, symbolic machine learning, statistical models, image/audio/video compression and retrieval, encouraging "new ideas, combining two or more areas, such as neurofuzzy, neuro-symbolic, neuro-evolutionary, neuro-symbolic, neuro-pattern recognition, fuzzy-evolutionary, evolutionary-symbolic, fuzzy-evolutionary, evolutionary-symbolic, fuzzy-symbolic, etc.'

The International Journal of Computational Intelligence (World Enformatika Society, since 2004) is a quarterly open access journal with a double-blind international review system. It is "focusing on theories, methods and applications in computational intelligence". There is no explanation what is meant by CI, just a statement that it deals "with any area of computational intelligence research". So far most papers in this journal are on various applications using a mixture of neural, fuzzy, and bio-inspired optimization methods. 
The International Journal of Computational Intelligence Research (Research India Publications, since 2005) is a free online journal. In description of its aims the connection with biology is stressed: "Computational intelligence is a well-established paradigm, where new theories with a sound biological understanding have been evolving. The current experimental systems have many of the characteristics of biological computers and are beginning to be built to perform a variety of tasks that are difficult or impossible to do with conventional computers." CI is considered to be heterogeneous field involving "such technologies as neurocomputing, fuzzy systems, probabilistic reasoning, artificial life, evolutionary algorithms, multi-agent systems etc." All of these of course performed using conventional computers.

The International Journal of Computational Intelligence Theory and Practice (Serials Publications, since 2006) "aims at publishing papers addressing theories, methods and applications in artificial neural networks, fuzzy systems, evolutionary computation, intelligent agents, hybrid systems and other areas of artificial intelligence”. No links to papers are provided, and no papers on classical AI have been published so far.

The Journal of Computational Intelligence in Bioinformatics (Research India Publications, 2006) covers "artificial intelligence and computational intelligence theory and their applications in bioinformatics". This journal tries to cover all "advances in computational molecular/structural biology, encompassing areas such as computing in biomedicine and genomics, computational proteomics and systems biology, and metabolic pathway engineering". The topics covered include many CI methods.

The IEEE Computational Intelligence Magazine (published by the IEEE Computational Intelligence Society, since 2006) covers "applications oriented developments, successful industrial implementations, design tools, technology reviews, computational intelligence education, and applied research". It also provides and overview of interesting CI topics in special issues.

The Computational Intelligence and Neuroscience (Hindawi Publishing, since 2007) is a new open access journal for "the interdisciplinary field of neural computing, neural engineering and artificial intelligence, where neuroscientists, cognitive scientists, engineers, psychologists, physicists, computer scientists, and artificial intelligence investigators among others can publish their work in one periodical that bridges the gap between neuroscience, artificial intelligence and engineering." This journal has a definite profile. "Artificial" probably means here "computational", and in most journal descriptions these words are treated as synonyms.

In the last year five new "computational intelligence" journals have been established. Unfortunately they all seem to be oriented towards methods rather than grand challenging problems to be solved. Some journals add fashionable topics like wavelet analysis, chaos, fractals, other go in the direction of AI, mentioning agents and reasoning as the main topics. The oldest CI journal happens to be a good old-fashioned AI in disguise.

For historical reasons these journals accept as valid CI topics selected statistical and mathematical techniques, such as Bayesian networks, probabilistic reasoning, rough sets and rough logic, basis set expansion methods for function approximation, support vector machines, kernel methods, or statistical natural language processing methods, while many other methods, including various statistical and logical approaches to clus- 
terization, classification, approximation, first and higher-order logic in reasoning, numerical optimizations techniques, approximation theory, or search techniques used to solve the same type of problems as the "valid CI methods", are beyond their scope. One can predict with confidence that many other journals called "Computational intelligence in xxx" will appear in the near future. Thus analysis of the topics covered by CI journals does not allow for clear understanding of what $\mathrm{CI}$ is or should be.

\section{CI books}

Perhaps books with "Computational Intelligence" will define the field better than journal descriptions. So far there are only a few textbooks with this title. The oldest one, Computational Intelligence - A Logical Approach [4], is a typical symbolic AI book focusing on logic and reasoning. The authors acknowledge that "Artificial intelligence is the established name for the field we have defined as computational intelligence", but think that "the term 'artificial intelligence' is a source of much confusion" and therefore propose to change the name, creating even greater confusion. In the first chapter they write: "Computational intelligence is the study of the design of intelligent agents. [...] The central scientific goal of computational intelligence is to understand the principles that make intelligent behavior possible, in natural or artificial systems". This could make their view of CI rather broad, because there are many approaches to analyze and model such systems. Unfortunately they focus only on reasoning as computation, and logic as the basis for reasoning, forgetting that symbols have first to be derived from real perceptions, and therefore pattern analysis cannot be avoided.

In the book Computational Intelligence for Decision Support similar definition is given: "Computational intelligence is the field of studying how to build intelligent agents" [5]. This obviously does not include most of what is discussed by CI community, presented at conferences, and published in CI journals. People with AI background evidently tend to see $\mathrm{CI}$ through the perspective of intelligent agents.

The book Computational Intelligence: An Introduction [6] defines CI as "the study of adaptive mechanisms to enable or facilitate intelligent behavior in complex and changing environments. As such, computational intelligence combines artificial neural networks, evolutionary computing, swarm intelligence and fuzzy systems". These are the main topics covered in the book, leaving aside many other CI topics.

Finally, the book Computational Intelligence: Principles, Techniques and Applications [7] contains a whole chapter in which the author tries to come up with a definition of $\mathrm{CI}$ by adding more and more methods to the core set that includes fuzzy, neural and evolutionary computations. This book covers also possibilistic reasoning, belief calculus, fuzzy Petri nets, and various combinations of these methods. It is perhaps the most ambitious attempt to define computational intelligence, discussing many exotic approaches, but still it falls short of covering all major tracks of any large conference on computational intelligence, for example it completely ignores kernel methods and basis set expansion networks.

Springer Studies in Computational Intelligence series has published already many books covering various aspects of CI. IEEE Computational Intelligence Society sponsors a book series on computational intelligence that is published by IEEE Press/Wiley. 
Several books apply CI techniques to specific areas, for example Computational Intelligence in Design and Manufacturing [8], Computational Intelligence in Software Quality Assurance [9], Computational Intelligence in Control Engineering [10], Computational Intelligence in Economics and Finance [11] and many others. They all tend to see computational intelligence as "a consortium of data-driven methodologies which includes fuzzy logic, artificial neural networks, genetic algorithms, probabilistic belief networks and machine learning" [11]

The Artificial Intelligence Portal in Wikipedia defines Computational intelligence (CI) as "a branch of the study of artificial intelligence. Computational intelligence research aims to use learning, adaptive, or evolutionary computation to create programs that are, in some sense, intelligent. Computational intelligence research either explicitly rejects statistical methods (as is the case with fuzzy systems), or tacitly ignores statistics (as is the case with most neural network research). In contrast, machine learning research rejects non-statistical approaches to learning, adaptivity, and optimization." According to this view $\mathrm{CI}$ is a part of AI focused on learning, but ignoring statistical methods. If CI is what computational intelligence experts do, this view is obviously false: kernel methods and Bayesian approaches are statistical learning techniques that are very much part of their bag of tools. AI experts have initially explicitly excluded all pattern recognition methods that $\mathrm{CI}$ community is very much interested in, so CI cannot be a part of classical AI.

\section{What should Computational Intelligence really be?}

For many CI experts biological inspirations are very important, but even if biology is extended to include all neural, psychological, and evolutionary inspirations this will only cover the main themes (neural, fuzzy and evolutionary) that the CI community works on. The whole Bayesian foundations of learning, probabilistic and possibilistic reasoning, other alternative approaches to handle uncertainty, kernel methods, information geometry and geometrical learning approaches, search algorithms and many other methods have little or no biological connections. Some neural methods are obviously more neural then others, with basis set function expansion methods having more to do with approximation theory than neurobiological inspirations. Why should only evolutionary algorithms be used if there are many specialized methods with proven convergence properties to solve specific optimization problems?

Physics studies nature and cannot be defined by its experimental or theoretical tools; the same is true for other branches of science. Computer science studies computable processes and information processing systems. What does computational intelligence study? CI studies problems for which there are no effective algorithms, either because it is not possible to formulate them or because they are NP-hard and thus not effective in real life applications. This is quite broad definition: computational intelligence is a branch of science studying problems for which there are no effective computational algorithms. Biological organisms solve such problems every day: extracting meaning from perception, understanding language, solving ill-defined computational vision problems thanks to evolutionary adaptation of the brain to the environment, surviving in a hostile environment. These problems require intelligence to solve but they 
may also be approached in different ways. Defining computational intelligence by the problems that the field studies has the advantage of removing the need to restrict the types of methods used for solution. Different fields obviously overlap with each other, and thus some problems will be of interest mainly for CI experts, while other problems will be of interests to experts in other fields. For example, optimization problems tackled by evolutionary, swarm, ant and other algorithms are of interest to operational research community. What problems are typical for computational intelligence?

A good part of $\mathrm{CI}$ research is concerned with low-level cognitive functions: perception, object recognition, signal analysis, discovery of structures in data, simple associations and control. Methods developed for this type of problems include supervised and unsupervised learning by adaptive systems, and they encompass not only neural, fuzzy and evolutionary approaches but also probabilistic and statistical approaches, such as Bayesian networks or kernel methods. These methods are used to solve the same type of problems in various fields such as pattern recognition, signal processing, classification and regression, data mining. Higher level cognitive functions are required to solve non-algorithmizable problems involving systematic thinking, reasoning, complex representation of knowledge, episodic memory, planning, understanding of symbolic knowledge. These problems are at present solved in a best way by AI community using methods based on search, symbolic knowledge representation, reasoning with frame-based expert systems, machine learning in symbolic domains, logics and linguistic methods. There is little overlap between problems solved using low and high-level mental functions, although they belong to the same broader category of non-algorithmizable problems.

From this point of view $\mathrm{AI}$ is a part of $\mathrm{CI}$ focusing on problems that require higher cognition and at present are easier to solve using symbolic knowledge representation. It is possible that other CI methods will also find applications to these problems in future. The main overlap areas between low and high-level cognitive functions are in sequence learning, reinforcement and associative learning, and distributed multi-agent systems. All tasks that require reasoning based on perceptions, such as robotics, automatic car driving, autonomous systems require methods for solving both low and high-level cognitive problems and thus are a natural meeting ground for AI experts with the rest of the CI community.

The idea that all intelligence comes from symbol manipulation has been perhaps misunderstood by AI community. Newell and Simon who originated this idea [12,13] wrote about physical symbols, not about symbolic variables. Physical symbols are better represented as multi-dimensional patterns representing states of various brain areas. Symbolic models of brain processes certainly do not offer accurate approximation for vision, control or any other problem that is described by continuous rather then symbolic variables. Approximations to brain processes should be done at a proper level to obtain similar functions. Symbolic dynamics [14] and extraction of finite state atomata from recurrent networks [15] may provide useful information on dynamical systems, and may be useful in modeling transition between low-to high level processes.

The division between low and high-level cognitive functions is only a rough approximation to the processes in the brain. Embodied cognition has been intensively studied in the last decade, and developmental ideas showing how higher processes emerge from 
the lower ones have been embraced by robotics people. Even in linguistics it is now commonly acknowledged that real meaning comes from body-based metaphors [16], and the same is true for such abstract areas as mathematics [17]. New CI methods that go beyond pattern recognition and help to solve AI problems may eventually be developed, starting from distributed knowledge representation, graphical methods and spreading activations networks. The dynamics of such models will probably allow for reasonable symbolic approximations, although this is still an open problem.

It is instructive to think about the spectrum of CI problems and various approximations needed to solve them. Neural network models are inspired by brain processes and structures at quite low single-neuron level, while symbolic AI models are inspired by processes at the highest level. The brain has a very specific modular and hierarchical structure, it is not one huge neural network. Perceptron model of a neuron has only one internal parameter, the firing threshold, and a few synaptic weights that determine neuron-neuron interactions. Single neurons probably influence brain information processing in quite insignificant way. Larger neural structures, such as microcircuits or neural cell assemblies, could also be used as basic processors for neural modeling. They have more complex internal states and more complex interactions between elements, but connectionist systems are not trying to approximate these process in any systematic way [18]. A network of networks, hiding the complexity of its processors in a hierarchical way, with different emergent properties at each level, will have progressively more internal knowledge and more complex interactions with other such systems. At the highest level models of whole brains with an infinite number of potential internal states and very complex interactions may be obtained. Discussion of such transition from neurons to brains and to societies is presented in [19].

Computational intelligence is certainly more than just the study of the design of intelligent agents, it includes also study of all non-algoritmizable processes that humans (and sometimes animals) can solve with various degree of competence, and the engineeering approaches to solve such problems using hardware and software systems. CI should not be treated as a bag of tricks without deeper foundations. Competition to solve CI problems using approaches developed in other fields should be invited. Knowledge and search-based methods should complement the core CI techniques in problems requiring reasoning. Goldberg and Harik [20] see computational intelligence more as a way of thinking about problems, calling for a "broader view of the scope of the discipline". They have analyzed limitations to progress in computational manufacturing design, finding the models of human behaviors to be most useful. Although this is certainly worthwhile defining clearly the problems that CI wants to solve and welcoming all methods that can be used in such solutions, independent of their inspirations, is even more important.

\section{Grand Challenges to Computational Intelligence}

A number of grand challenges for AI has been formulated, starting with the famous Turing Test for machine intelligence, This requires a very-large knowledge base and efficient retrieval of structures. While the $\mathrm{CyC}$ project [21] has created huge knowledge base manually coding it over a period of more than 30 years the retrieval mech- 
anisms that it offers are too inefficient to use it in large-scale dialog systems. A grand challenge for $\mathrm{CI}$ community is to propose more efficient knowledge representation and retrieval structures, perhaps modeled on the associative memory of the brain, perhaps using different knowledge representations for different purposes [22]. Vector and similarity-based models cannot yet replace complex frames in reasoning processes. Semantic networks, although in principle could provide efficient association and inference mechanisms, have never been used on a large scale.

Feigenbaum [23] proposed a reasoning test which should be simpler for computers than the Turing Test. Instead of a general dialog that has to be based on extensive knowledge of the world, this test is based on the expert knowledge in a narrow domain. Reasoning in some field of mathematics or science by human expert and artificial system should be evaluated by another expert in the same field who will pose problems, questions, and ask for explanations. This could be achieved with super-expert systems in various domains, giving some measures of progress towards intelligent reasoning systems. The World Championship for 1st Order Automated Theorem Proving organized at the Conference on Automated Deduction (CADE) could be organized not only between computers, but could also involve humans, although much longer time to complete the proofs may be required. Other grand AI challenges [23] are concerned with large-scale knowledge bases, bootstraping on the knowledge resources from the Internet and creating semantic Internet. The 20 -questions game could also be a good challenge for AI, much easier than the Turing test, requiring extensive knowledge about objects and their properties, but not about complex relations between objects. In fact some simple vector-space techniques may be used to play it [22], making it a good challenge not only for AI, but also for the broader CI community.

What would be a good grand challenge for non-AI part of computational intelligence? This has been the subject of a discussion panel on the challenges to the $\mathrm{CI}$ in the XXI century, organized at the World Congress on Computational Intelligence in Anchorage, Alaska, in 1998. The conclusion was that a grand challenge for CI is to build an artificial rat, an artificial animal that may survive in a hostile environment. The intermediate steps require solution to many problems in perception, such as object recognition, auditory and visual scene analysis, spatial orientation, memory, motor learning, behavioral control, but also some reasoning and planning. The ultimate challenge may be to build not only an animal, but a human-like system that in addition to survival will be able to pass the Turing test.

Imagine the future in which superintelligence based on some form of computations has been realized. In the long run everything seems to be possible, but what we would like it to do and like it to be? Computational intelligence should be human-centered, helping humans not only to solve their problems, but also to formulate meaningful goals, leading to a true personal fulfillment. It should protect us starting from birth, not only monitoring the health hazards, but also observing and guiding personal development, gently challenging children at every step to reach their full physical as well as mental potential. It should be a technology with access to extensive knowledge, but it also should help humans to make wise decisions presenting choices and their possible consequences. Although it may seem like a dangerous utopia perhaps deeper understanding of developmental processes, cognitive and emotional brain functions, 
real human needs, coupled with a technology that can recognize behavioral patterns, make sense of observations, understand natural language, plan and reason with extensive background knowledge, will lead to a better world in which no human life is wasted. Intelligence with wisdom is perhaps an ultimate goal for human-oriented science. Such utopia is worth dreaming of, although we are still very far from this level (see some speculations on this topic in [24-26]).

A long-term goal for computational intelligence is to create cognitive systems that could compete with humans in large number of areas. So far this is possible only in restricted domains, such as recognition of specific patterns, processing of large amount of numerical information, memorization of numerous details, high precision control with small number of degrees of freedom, and reasoning in restricted domains, for example in board games. Brains are highly specialized in analysis of natural patterns, segmentation of auditory and visual scenes, and control of body movements, mapping perceptions to actions. Despite great progress in computational intelligence artificial systems designed to solve lower level cognitive functions are still far behind the natural ones. Situation is even worse when higher-level cognitive functions, involving complex knowledge structures necessary for understanding of language, reasoning, problem solving or planning, are considered. Human semantic and episodic memory is vastly superior to the most sophisticated artificial systems, storing complex memory patterns and rapidly accessing them in an associative way.

So far CI understood as a collection of different methods had no clear challenges of the AI magnitude. Improving clusterization, classification and approximation capabilities of CI systems is incremental and there are already so many methods that it is always possible to find alternative solutions. At the technical level fusion of different CI techniques is considered to be a challenge, but attempts to combine evolutionary and neural methods, to take just one example, have a long history and it is hard to find results that are significantly better than those achieved by competing techniques. The challenge is at the meta-level, to find all interesting solutions automatically, especially in difficult cases. Brains are flexible, and may solve the same problem in many different ways. Different applications - recognition of images, handwritten characters, faces, analysis of signals, mutimedia streams, texts, or various biomedical data - usually require highly specialized methods to achieve top performance. This is a powerful force that leads to compartmentalization of different CI branches. Creation of meta-learning systems competitive with the best methods in various applications is a great challenge for CI.

If we recognize that CI should be defined as the science of solving non-algorithmizable problems using computers or specialized hardware the whole field will be firmly anchored in computer and engineering sciences, many technical challenges may be formulated, and important biological sources of inspiration acknowledged. Focusing on problems instead of tools will enable competition with other methods for various applications, facilitating real progress towards more difficult problems. It should also allow for greater integration of CI and AI communities working on real-world problems that require integration of perception with reasoning. Broad foundations for $\mathrm{CI}$ that go beyond pattern recognition need to be constructed, including solving problems related to the higher cognitive functions (see [27], this volume). Inspirations drawn from cogni- 
tive and brain sciences, or biology in general, will continue to be very important, but at the end of the road CI will become a solid branch of science on its own standing.

Acknowledgement. I am grateful for the support by the Polish Committee for Scientific Research, research grant 2005-2007.

\section{References}

1. H.A. Simon, Artificial Intelligence: Where Has it Been, and Where is it Going? IEEE Transactions on Knowledge and Data Engineering 3(2): 128-136, 1991.

2. A.K. Mackworth, The Coevolution of AI and AAAI, AI Magazine 26(4): 51-52, 2005.

3. J. C. Bezdek, What is computational intelligence? In: Computational Intelligence Imitating Life, pp. 1-12, IEEE Press, New York, 1994.

4. D. Poole, A. Mackworth and R. Goebel. Computational Intelligence - A Logical Approach. Oxford University Press, New York, 1998.

5. Z. Chen, Computational Intelligence for Decision Support. CRC Press, Boca Raton, 2000.

6. A.P. Engelbrecht, Computational Intelligence: An Introduction. Wiley, 2003.

7. A. Konar, Computational Intelligence: Principles, Techniques and Applications. Springer 2005.

8. A. Kusiak, Computational Intelligence in Design and Manufacturing. Wiley-Interscience, 2000.

9. S. Dick and A. Kandel, Computational intelligence in software quality assurance. Series in Machine Perception and Artificial Intelligence, Vol. 63, World Scientific 2005.

10. R.E. King, Computational intelligence in control engineering, Marcel Dekker Inc., NY, 1999.

11. S.H. Chen, P. Wang, and P.P. Wang Computational Intelligence in Economics and Finance. Advanced Information Processing Series, Springer 2006.

12. A. Newell and H.A. Simon, Computer science as empirical enquiry: Symbols and search. Communications of the ACM 19(3), 113-126, 1976.

13. A. Newell, Unified Theories of Cognition. Cambridge, MA: Harvard University Press 1990.

14. D. Lind, B. Marcus, Symbolic Dynamics and Coding, Cambridge University Press, 1995.

15. H. Jacobsson, Rule extraction from recurrent neural networks: A taxonomy and review. Neural Computation, 17(6), 1223-1263, 2005.

16. G. Lakoff, M. Johnson. Metaphors We Live By. University of Chicago Press, 2nd ed, 2003.

17. G. Lakoff, R. Núnez, Where Mathematics Comes From: How the Embodied Mind Brings Mathematics into Being. Basic Books 2000.

18. A. Clark, R. Lutz (eds), Connectionism in Context. Springer-Verlag, Berlin, 1992.

19. W. Duch and J. Mandziuk, Quo Vadis Computational Intelligence? In: Machine Intelligence: Quo Vadis? Advances in Fuzzy Systems - Applications and Theory (eds. P. Sincak, J. Vascak, K. Hirota), World Scientific 2004.

20. D.E. Goldberg and G. Harik, A Case Study in Abnormal CI: The Design of Manufacturing and Other Anthropocentric Systems. International J. Computational Intelligence and Organizations, 1, 78-93, 1996.

21. D. Lenat and R. V. Guha, Building Large Knowledge-Based Systems: Representation and Inference in the Cyc Project. Addison-Wesley 1990.

22. J. Szymanski, T. Sarnatowicz and W. Duch, Towards Avatars with Artificial Minds: Role of Semantic Memory. Journal of Ubiquitous Computing and Intelligence (in print)

23. E.A. Feigenbaum, Some Challenges and Grand Challenges for Computational Intelligence. Journal of the ACM 50(1), 32-40, 2003.

24. R. Kurzweil. The age of spiritual machines: When computers exceed human intelligence. Penguin, New York, NY, 1999. 
25. J. McCarthy, The Future of AI-A Manifesto. AI Magazine 26, 39-40 2005

26. L. Perlovsky. Knowledge Instinct. Basic Books, 2006.

27. W. Duch, Towards comprehensive foundations of computational intelligence. In: Duch W, Mandziuk J, Eds, Challenges for Computational Intelligence. Springer 2007 